\title{
Graph Radial Layout
}

National Cancer Institute

\section{Source}

National Cancer Institute. Graph Radial Layout. NCI Thesaurus. Code C85852.

A type of graph that uses a focus node with other nodes arranged in concentric circles around the focus to illustrate the central node's degree of connectivity to the other nodes. 